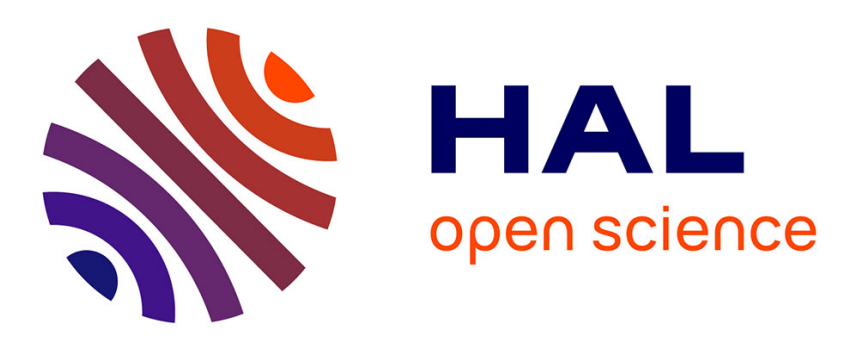

\title{
Des normes financières islamiques et de leurs circulations en France et en Grande Bretagne
}

Bochra Kammarti

\section{To cite this version:}

Bochra Kammarti. Des normes financières islamiques et de leurs circulations en France et en Grande Bretagne. Archives de Sciences Sociales des Religions, 2017, pp.255 - 280. 10.4000/assr.29671 . hal03343539

\section{HAL Id: hal-03343539 \\ https://hal.science/hal-03343539}

Submitted on 14 Sep 2021

HAL is a multi-disciplinary open access archive for the deposit and dissemination of scientific research documents, whether they are published or not. The documents may come from teaching and research institutions in France or abroad, or from public or private research centers.
L'archive ouverte pluridisciplinaire HAL, est destinée au dépôt et à la diffusion de documents scientifiques de niveau recherche, publiés ou non, émanant des établissements d'enseignement et de recherche français ou étrangers, des laboratoires publics ou privés. 


\section{Archives de sciences sociales des religions}

179 | Juillet-septembre 2017

Sociologies catholiques. Référentiel islamique

\section{Des normes financières islamiques et de leurs circulations en France et en Grande Bretagne}

Islamic financial norms and their circulation in France and Great Britain Normas financieras islámicas y su circulación en Francia y Gran Bretaña

Bochra Kammarti

\section{OpenEdition}

Journals

Édition électronique

URL : http://journals.openedition.org/assr/29671

DOI : 10.4000/assr.29671

ISSN : $1777-5825$

Éditeur

Éditions de l'EHESS

Édition imprimée

Date de publication : 1 septembre 2017

Pagination : $255-280$

ISBN : 978-2-7132-2694-6

ISSN : 0335-5985

Référence électronique

Bochra Kammarti, «Des normes financières islamiques et de leurs circulations en France et en

Grande Bretagne », Archives de sciences sociales des religions [En ligne], 179 | Juillet-septembre 2017, mis en ligne le 01 septembre 2019, consulté le 05 janvier 2021. URL : http://journals.openedition.org/ assr/29671; DOI : https://doi.org/10.4000/assr.29671 


\section{Des normes financières islamiques et de leurs circulations en France et en Grande-Bretagne}

La différence islamique en matière de financements s'observe par un ensemble de prescriptions et de normes issues de la sharîa - du Coran et de la Sunna (tradition prophétique) - se rapportant aux transactions, mu'âmalât. Ces prescriptions islamiques sont à l'origine d'un ensemble de contrats et de modes de financements qui unissent, de manière plus ou moins homogène, le champ de la finance islamique. Leur utilisation génère une réflexivité juridique qui s'est traduite par la production d'un corpus de références sémantiques, normatives et contractuelles. Avec leur circulation, en particulier en Europe ${ }^{1}$, les instruments de la finance islamique sont amenés à être pensés et définis dans leurs dissensions avec les normes séculières locales. La sociogenèse de la finance islamique a été mise en œuvre par la coopération d'acteurs islamiques et séculiers (jurisconsultes musulmans, avocats, juristes, instances de réglementations financières, professionnels financiers, etc.), des traducteurs de normes et de pratiques, qui sont à leur tour les vecteurs de la circulation et de la dissémination de ces normes.

Cette sociogenèse témoigne de résistances liées à l'apparition du référentiel islamique au sein de contextes nationaux codifiés par des monopoles d'usages (financiers, linguistiques et juridiques), mais aussi des dynamiques de créations sociales qui permettent le dépassement des contraintes imposées par les normes, les valeurs, les systèmes de référence linguistiques et juridiques islamiques et séculiers. Dans cette dialectique, les espaces séculiers européens sont interpénétrés par le référentiel islamique (Göle, 2005) et sont amenés à se redéfinir

\footnotetext{
1. Si les premières expériences ont lieu dans les années 1950 et 1960 au Pakistan, en Égypte et en Malaisie, le développement de l'industrie financière islamique remonte aux années 1970 avec la création de la Banque islamique de développement (BID) en 1973 à Djeddah. L'Islamic Banking System est la première institution financière islamique en Europe, créée en 1978 au Luxembourg. Des banques conventionnelles européennes ont ouvert des succursales islamiques (Islamic windows) et en 2004, la première banque islamique de détail nationale européenne à vu le jour en Grande Bretagne (l'Islamic Bank of Britain) ainsi que trois autres banques d'investissement. La pénétration de la finance islamique en Europe correspond à la troisième phase de développement du système bancaire islamique (Ruimy, 2008).
} 
ou à réitérer leur différence par «des opérations d'évaluation et de jugement qui permettent [...] de recomposer la communauté morale nationale» (Fassin, 2012). Nous procèderons à l'analyse de ces tensions et des dénouements à travers, d'une part, la comparaison de la réglementation du contrat islamique de la murâbaha $a^{2}$ par les institutions séculières britanniques et françaises et d'autre part, la comparaison des décisions juridiques relatives aux règles de droit applicables en cas de litige devant les tribunaux.

Au-delà de la question de savoir si la finance islamique et la finance conventionnelle convergent, se complémentent, ou au contraire, s'opposent, l'objet de cet article porte sur des lieux de rencontre, européens, des champs sociaux où émergent la normativité islamique en matière de financements et les conflits qui résultent de cette rencontre. Ces conflits montrent une double tendance qui peut paraître paradoxale: alors que le référentiel islamique pénètre et s'enracine dans ces lieux - qu'ils soient physiques ou symboliques - il sert aussi à dessiner et délimiter les frontières morales - historiques, valeurs ou juridiques - de la communauté nationale. C'est en ce sens que la définition apportée par Didier Fassin des économies morales guide cette analyse ${ }^{3}$. Cette économie morale se lie à la (re)production de valeurs, de normes, de traditions et d'histoires qui construisent des frontières immatérielles aux communautés nationales dans leur rencontre avec l'islam.

\section{La production et la répartition de normes relatives au champ financier islamique}

\section{Sharîa, fiqh al-mu'âmalât, sharîa board et fatwas}

Sharîa trouve sa racine dans le mot shar', qui signifie "chemin », "voie ». Dans l'Encyclopédie de l'islam, il "désigne les lois et règlements régissant l'existence des Musulmans, dérivées en principe du Coran et des hadiths » (Calder, 1998 : 331). Dans cette acception du terme, elle renvoie principalement aux aspects normatifs et juridiques de la religion. Mais sharîa peut aussi couvrir

2. La murâbaha est une opération d'achat-revente d'un bien: un intermédiaire financier achète le bien à un fournisseur et le revend à son client moyennant une marge bénéficiaire. Du mot ribh qui signifie gain ou profit, cet instrument représente $70 \%$ des financements du secteur de la finance islamique (Saïdane, 2009). Son origine est discutée : l'économiste Ruimy (2008) situe son apparition en 1995 dans le secteur de l'aviation pour l'achat de kérosène entre la Pakistan International Airlines et l'Islamic Investment Company of the Gulf; l'islamologue Saeed (1996) indique que cette transaction n'existe pas dans le Coran et dans les hadiths mais qu'elle se pratiquait depuis l'époque de Médine, une thèse qui contredit celle d'Al Kaff (1986) qui nie l'existence de la murâbaha au temps du prophète et de ses compagnons. Sa terminologie existe au moins depuis l'époque d'Ibn Rushd (1126-1198) dans son œuvre Bidâyat al-mujtahid wa nihâyat al-muqtasid et dans le Livre du Travail et des moyens de subsistance, Al Ghazalî (1058-1111) décrit en détail ce qui est licite et illicite concernant la vente et l'usure.

3. L'anthropologue «considère l'économie morale comme la production, la répartition, la circulation et l'utilisation des émotions et des valeurs, des normes et des obligations dans l'espace social » (Fassin, 2012:37) 
une dimension spirituelle et se rapporter à l'éthique islamique, en particulier au message du Prophète Muhammad (Calder, 1998). Elle renvoie alors plus à des questions philosophiques, théologiques et morales qu'à des aspects normatifs (Ebnou, 2014). Son usage dans le champ social financier islamique européen - publications, conférences et entretiens que nous avons menés procède d'un jeu de langage (Dupret, 2012, 2014). Lorsqu'il s'agit d'expliquer la finance islamique et de la différencier de la finance conventionnelle, on se rapporte aux prescriptions islamiques - au licite, halal, et à l'illicite, haram. Lorsqu'il s'agit de faire converger finance islamique et finances éthiques, ISR, etc. avec une volonté et le souci d'œuvrer pour une finance plus juste, les locuteurs insistent plutôt sur sa dimension éthique. Ils se rapportent alors aux valeurs - la recherche de l'équité, de l'intérêt général, l'économie du réel, etc. Dans cet article, il renvoie le plus souvent à la fois aux prescriptions et à l'éthique islamique.

L'éthique et les prescriptions islamiques - le licite et l'illicite - issues de la sharîa se rapportant aux transactions économiques et financières, sont traitées dans le fiqh al-mu'âmalât ${ }^{4}$. Le fiqh regroupe l'ensemble des interprétations et avis juridiques des jurisconsultes musulmans établis à partir du Coran et de la Sunna (tradition prophétique) et contenus dans la jurisprudence musulmane. Il constitue le fruit de l'effort d'interprétation, l'ijtihâd, du Coran et de la Sunna, il est une œuvre humaine sujette à la subjectivité de celui qui interprète ${ }^{5}$. La sharîa d'un côté (source première) et le fiqh d'un autre (sources secondaires) servent de références normatives qui permettent aux jurisconsultes musulmans d'émettre un avis, une fatwa ${ }^{6}$. Dans le champ financier islamique, les sharîa scholars, jurisconsultes musulmans, certifient la licité des transactions et des produits financiers islamiques par l'édition de fatwas. Ces derniers doivent à la fois maîtriser les connaissances en matière de religion, mais aussi dans les domaines financiers et économiques. Ils sont engagés pour une durée déterminée par une institution financière islamique, privée ou publique, ou par des Islamic windows ${ }^{7}$ et siègent au sein de sharîa

4. On distingue les traités de fiqh al-'ibadāt qui se rapportent aux relations des hommes à Dieu et portent sur les obligations rituelles relatives aux cinq piliers de l'islam - la chahâda (profession de foi), la salât (la prière), la zakât (aumône légale), ramadân (le jeûne) et le hajj (le pèlerinage) - des traités de fiqh al-mu'âmalât qui se rapportent aux relations entre les hommes. Cette partie $\mathrm{du}$ fiqh concerne surtout les relations familiales (mariage, divorce, etc.), mais aussi, la finance, "des sujets qui n'ont été que faiblement balisés par la doctrine mais qui trouvent dans le Texte sacré une disposition justifiant de les réglementer comme, par exemple, l'interdiction de l'usure » (Dupret, 2014: 15).

5. Le fiqh repose sur les principes du raisonnement par analogies (qiyâs), sur la coutume ('urf) et le droit coutumier ('âdât). Ces jugements doivent faire l'objet d'un consensus de la communauté $(i j m \hat{a})$. Le sunnisme distingue quatre écoles de fiqh: le hanafisme, le malékisme, le chafiisme et le hanbalisme.

6. Une fatwa est une technique particulière de la consultation qui se présente sous forme de question/réponse (Dupret, 2014).

7. Traduit en français par "fenêtre ou guichet islamique ", une Islamic window est un département ou succursale d'une banque conventionnelle qui offre des produits et des services financiers 
boards ${ }^{8}$ - organe collégial qui assure la conformité des transactions aux principes juridico-éthiques de l'islam (Ould Sass, 2010). Cet organe consultatif n'émet qu'un avis, mais il bénéficie d'un pouvoir symbolique important: il contribue à la «construction de confiance auprès d'une clientèle sensible à la notion de légitimité éthico-religieuse» (Ould Sass, 2010).

\section{La différence islamique en matière de financements}

La finance islamique se distingue de la finance conventionnelle par le respect de prescriptions liées aux transactions. Ces prescriptions sont issues de la sharîa, Coran et Sunna qui «(guident) le comportement économique du «bon » musulman, en lui précisant ce qui est licite et ce qui est illicite du point de vue religieux" (Chapellière, 2009: 23). Plus précisément, elle se définit par l'interdiction du rib $\hat{a}^{9}$ - intérêt - l'interdiction de spéculer - maysir ${ }^{10}$ d'agir dans l'incertitude - gharar ${ }^{11}$ - l'interdiction de financer des secteurs ou de consommer des produits illicites ${ }^{12}$ - harâm - l'obligation de partager

islamiques. Plusieurs banques conventionnelles européennes ont ouvert des Islamic windows: HSBC Amanah, BNP Nejma, UBS Islamic Finance, Citi Islamic Investment Bank (CIIB), Lloyds TSB, etc. 8. Son usage en anglais est le plus courant, y compris en France. Son origine remonte à l'institution de la hisba, autorité éthique de marché qui bénéficiait d'un pouvoir moral et d'un mandat de supervision sur la vérification des mesures, la prévention des fraudes et la sensibilisation aux bonnes pratiques commerciales (Ould Sass, 2010). Les sharîa boards sont composés selon l'AAOIFI (Accounting and Auditing Organization for Islamic Financial Institutions) - association internationale qui encadre l'industrie financière islamique - d'au moins trois jurisconsultes (sharîa scholars), nommés et mandatés pour une durée déterminée par l'Assemblée générale ou le Conseil d'administration de l'institution financière. Les sharîa scholars peuvent siéger dans plusieurs comités et dans différentes institutions financières à la fois.

9. Entre autres versets: Sourate II, verset 275-279; sourate III, verset 130; sourate IV, verset 160-161 et sourate xxx, verset 39. L'interdit est rapporté également par plusieurs hadiths issus de la Sunna. Issu du mot rabâ', ribâa' signifie « augmentation, addition, expansion ou croissance » (Chapra, 2006), il se rapporte à la notion d' 'accroissement, comme terme technique, usure et intérêt, de même que d'une façon générale tout avantage pécuniaire illégitime sans équivalent de service rendu " (l'Encyclopédie de l'islam, 1995: 508). Dans la casuistique musulmane ribâa' se rapporte à tout gain illicite (Müller, 2010). Le Dictionnaire du Coran (2007), comme certaines traductions du Coran (Masson, 1967), traduisent ribâa' par « usure », alors qu'elle est communément entendue par les jurisconsultes musulmans par "intérêt» ou toute forme de rémunération de l'argent par le seul facteur de l'écoulement du temps. Pour Umer Chapra, économiste islamique, ribầ' se rapporte tant au ribâa' al-nasi'ab-l'intérêt perçu sur les prêts - qu'au ribâ' al-fadl-excès perçu d'une transaction (Chapra, 2006). Dans le droit financier et commercial islamique, l'interdit du ribâ" est "compris comme l'interdit du prêt à intérêt » (Dictionnaire du Coran, 2007: 829). 10. Sourate v, verset 90 et Sourate II, verset 219. Le maysir ou qimâr vise toute forme de contrat qui dépend d'un événement aléatoire. Il était à l'origine un jeu à mises des parties dépecées d'une pièce de bétail qui se pratiquait avec des fléchettes. Est-ce le fait de miser sur quelque chose ou l'aléa que représente le jeu qui est interdit? Les savants musulmans débattent encore aujourd'hui sur ce point (Éric Chaumont, «Jeu, jeu de hasard ", Dictionnaire du Coran, Mohammed Ali Amir Moezzi (dir.), Robert Laffont, p. 441-443).

11. Sourate v, versets 90 et 91 .

12. L'armement, le vin, le porc, les casinos, la prostitution, etc. 
les pertes et les profits ${ }^{13}$ et l'obligation de reverser un impôt légal à des œuvres charitables, la zakât - $3^{\mathrm{e}}$ pilier de l'islam - et l'obligation d'adosser la transaction à un actif tangible.

Ces prescriptions différencient la finance islamique de la finance conventionnelle sans être incompatibles avec cette dernière. En effet, la finance islamique offre des produits et des services financiers qui ont des équivalents en finance conventionnelle ${ }^{14}$. Pour autant, elle se distingue par le fait qu'elle se fonde sur des prescriptions morales. Porteuse d'un projet éthique, d'un humanisme ${ }^{15}$ (De Courcelles, 2014), elle doit tenir compte des aspects humains, sociaux et environnementaux et vise, à ce titre, la distribution équitable de la richesse et des revenus, l'absence de gaspillage, le développement de l'économie réelle et l'augmentation de l'emploi (Martens, 2001, Chapellière, 2009, Guéranger, 2009, Martin, 2012, De Courcelles, 2014). Dans ce sens, les auteurs la présentent comme une «finance alternative»: une solution financière éthique et socialement responsable au même titre que le sont les fonds éthiques et les fonds d'investissement socialement responsable (ISR) (Guéranger, 2009, Martin, 2012, Roux, 2012).

L'industrie financière islamique n'émerge pas comme un contre espace du système financier et ne développe pas à ce titre de forme de résistances, bien au contraire, elle tend à travers son offre de produits et services à pénétrer le système financier par la voie du «développement durable». En France particulièrement, elle émerge sous la forme d'un discours éthique apportant des solutions à l'hyper-rationalité du système financier conventionnel en crise. Elle n'est donc pas une forme concurrente, mais œuvre à être un secteur financier du capitalisme socialement responsable. Dr Ahmad Mohamed Ali, Président de la Islamic Development Bank Group (BID), a rappelé lors de son discours prononcé au $3^{\text {e }}$ Forum français de finance islamique que "l'effort conjoint des institutions islamiques vise à intégrer la finance islamique dans le système financier mondial ${ }^{16}$.

Seule l'interdiction du rib $\hat{a}^{17}$, intérêt, confronte prescriptions islamiques et normes conventionnelles: dans un cas, il est illicite, harâm; dans l'autre, un

13. La finance islamique engage les partenaires à partager les pertes et les bénéfices. De ce fait, elle peut être considérée comme une "finance participative». D’ailleurs, en Turquie on préfère cette dernière dénomination à celle de «finance islamique» (Chapellière, 2009).

14. Voir l'ouvrage de Dhafer Saïdane dans lequel l'auteur décrit les différents contrats islamiques et leurs équivalents en finance conventionnelle (Saïdane, 2009).

15. Pour la philosophe, l'humanisme place l'homme dans une constante dialectique entre liberté et responsabilité, il est, dans ce sens, éthique et vise la juste redistribution des richesses, matérielles et immatérielles. L'humanisme spirituel peut ainsi constituer l'horizon ultime d'une «éthique économique» (De Courcelles, 2014).

16. Traduction de: "The joint effort of the Islamic institutions aims at mainstreaming Islamic finance in the global financial system", Dr. Ahmad Mohamed Ali, le 9 décembre 2009 à Paris. 17. En Europe, cet interdit est le plus cité par les jurisconsultes, les acteurs de la finance islamique et les usagers musulmans qui souhaitent entreprendre des projets professionnels et personnels en conformité aux prescriptions islamiques. Son interdit proscrit l'emprunt conventionnel et rend difficile le financement des projets en conformité à l'éthique islamique (Kammarti, 2015b : 168-190). 
monopole d'usage ${ }^{18}$. Lorsqu'une personne souhaite financer un projet personnel ou professionnel, la pratique courante consiste à emprunter un capital financier afin de réaliser son projet. En finance conventionnelle, le monopole d'usage consiste à faire payer une somme supplémentaire en fonction du temps écoulé, un «taux d'intérêt ». En finance islamique, cette pratique est interdite en raison de la proscription du ribâ'. Pour les musulmans soucieux du respect de la sharîa dans leurs pratiques au quotidien, l'interdiction du ribâa' implique l'usage de modes alternatifs de financement au prêt à intérêt ${ }^{19}$.

\section{La création du Shari'a standards for Islamic financial institutions}

L'essor du secteur financier islamique depuis les années 1970 et les contraintes établies par la disparité des interprétations des normes relatives au champ financier ont conduit des fuqahâ', des jurisconsultes musulmans, à œuvrer en commun à la production d'un corpus normatif standardisé de fatwas et de contrats propre au champ financier islamique. Sous la directive de l'AAOIFI ${ }^{20}$ et du Comité de Fatwa et d'Arbitrage, une commission de consultants a été créée en mars 1999 afin d'étudier les règles islamiques s’appliquant aux transactions conformes à la sharîa. Suite à des consultations restreintes entre jurisconsultes et une discussion publique élargie incluant des professionnels des métiers liés à la finance, les résultats de ce travail ont abouti à la publication

18. Voir, sur la pratique de l'intérêt et le processus par lequel elle est devenue un monopole d'usage en Europe (Kammarti, 2015a : 149-155).

19. En 2004, en Grande-Bretagne, une enquête quantitative Demand for Islamic financial services in the United Kingdom: Chasing or mirage? menée par Humayon Dar, professeur d'économie de Loughborough University, établit que 5\% des personnes interrogées ne consomment pas de produits et de services financiers basés sur l'intérêt en raison de leur foi, une grande majorité $(75 \%)$ se satisfait des services financiers conventionnels. En France, selon un rapport établi par l'AIDIMM et l'IFAAS en 2011, Finance islamique, quels marchés et quelles opportunités pour les banques de détails sur la base d'une enquête menée par l'institut de sondage IFOP, plus de la moitié des personnes interviewées seraient intéressées par des produits de financement conforme à l'éthique islamique, mais un faible pourcentage de personnes n'ont pas souscrit d'emprunt et n'ont pas l'intention de le faire pour motif religieux (principalement à cause de l'interdiction du ribấ) (12\%). Parmi les entretiens qualitatifs menés, la plupart des musulmans pratiquants avec qui nous nous sommes entretenus ont eu recours à des emprunts conventionnels ou ont l'intention de le faire s'ils en éprouvent le besoin. Il a fallu sélectionner des profils, en particulier des «entrepreneurs islamiques ", pour observer l'usage de moyen de financements alternatifs aux emprunts conventionnels dans le cas du financement de leurs projets professionnels.

20. L'Accounting and Auditing Organization for Islamic Financial Institutions est une association internationale à but non lucratif créée en 1990 par la coopération de la Banque Islamique de Développement (BID), d'institutions financières et des institutions du fiqh islamique. Elle encadre l'industrie financière islamique et ses institutions par la mise en place de normes et de standards qui respectent les règles de droits islamiques. En 2002, un autre organe international de normalisation des pratiques liées à l'industrie financière islamique a été crée à Kuala Lampur, en Malaisie, l'Islamic Financial Service Board (IFSB), il a pour vocation comme l'AAOIFI, de «promouvoir et renforcer la solidité et la stabilité de l'industrie des services financiers islamiques en émettant des normes prudentielles mondiales et des principes directeurs pour l'industrie ". Ces deux organismes internationaux sont les principaux référents en matière de normes, de pratiques et de la conformité à la sharîa des financements islamiques. 
de normes standardisées régissant les transactions dans la conformité des règles de droit islamique ${ }^{21}$.

Ce corpus normatif a été publié en 2007 en arabe et traduit en anglais en 2010 sous le nom de Shari'a (sic) standards for Islamic financial institutions ${ }^{22}$. Il répertorie les différents modes de financements islamiques et expose à la fois les procédures licites relatives aux échanges économiques et les sources qui légitiment ces procédés. Comme un répertoire ${ }^{23}$, il articule, dans un registre linguistique d'interdit et de permissivité, les outils contractuels, les modes opératoires et les manières de faire des affaires en conformité avec la sharîa. Ce guide a vocation à établir une connaissance qui permette de créer des valeurs, des pratiques et des instruments transférables à d'autres contextes réglementaires (Maurer, 2002). Il peut par exemple en Europe servir de référence contractuelle ou être cité par les juges et les arbitres en cas de litiges (Affaki, 2008). Son utilisation n'est cependant pas systématique: il est surtout connu des professionnels, financiers et juridiques, des institutions financières islamiques ou conventionnelles et des sharîa scholars. Les cas de litiges en Grande-Bretagne montrent qu'il est le plus souvent ignoré des juges et sa publication n'évite pas différentes interprétations et fatwas relatives aux transactions économiques.

\section{Répartition des normes en Europe}

La constitution d'un répertoire contractuel propre à la finance islamique émerge en parallèle d'interprétations normatives et d'éditions de fatwas diverses, parfois même contraire à l'effort de légitimation et de consolidation du secteur financier islamique, et ce en particulier, parce qu'elles sont formulées de manière à répondre à des contraintes immédiates, dans des espaces différents. "Cette dynamique (la nécessaire révision de la sharîa) se traduit le plus souvent par l'examen détaillé des diverses techniques, des théories et des pratiques juridiques islamiques qui permettent de moduler, voire de réformer l'énoncé de la sharîa en fonction du cadre particulier et du vécu des musulmans dans les sociétés occidentales. Se dessinent alors trois types de positionnement à la fois distincts et complémentaires : la reformulation

\footnotetext{
21. Shari'a standards for Islamic financial institutions, Accounting and Auditing Organization for Islamic Financial Institutions, version anglaise 2010 (1432H), version arabe 2007 (1428H). 22. Il sera traduit en français en 2013 sous l'égide de Paris Europlace: Finance islamique, les normes de conformité de l'AAOIFI, Eska, coll. "Gestion financière ", 2013.

23. Il constitue un répertoire normatif (Dupret, 2000), c'est-à-dire "un ensemble regroupant ces ressources formelles que sont les règles autour d'un principe de légitimité. [...] La notion de répertoire normatif permet [...] de faire état de principes de justifications multiples, mobilisées en fonction de situations circonstancielles, les contextes d'interaction [...]. Le répertoire n'est pas tant déterminé par la circonstance que par le jeu argumentatif choisi par son utilisateur en fonction des circonstances» (Dupret, 2000: 13). Mais il n'est pas seulement discursif et argumentatif, comme un idéal-type, il sert aussi de modèle contractuel aux acteurs qui peuvent s'y référer pour élaborer une opération économique.
} 
de la charia; la relativisation du poids de la norme et même l'abandon pur et simple de celle-ci» (Frégosi, 2012: 71).

L'Europe est en ce sens un lieu privilégié pour observer ces modulations et cette plasticité de la norme. Nous exposerons dans cet article une sélection d'avis juridiques relative à la transaction de murâbaha - transaction la plus fréquente mais aussi la plus controversée parmi les jurisconsultes. Pour certains, elle constitue une hîla, une ruse qui consiste à cacher l'intérêt par une marge bénéficiaire, pour d'autres, il s'agit d'un outil de transition nécessaire vers un système financier islamique idéal, en particulier dans des contextes où le monopole d'usage des prêts à intérêts ne permet pas aux musulmans de réaliser leurs projets personnels ou professionnels. Ces avis juridiques ont été exprimés ou traduits en français et sont accessibles aux musulmans en France via Internet. Cette sélection ne se veut pas exhaustive, elle a pour objectif d'illustrer la disparité des avis juridiques et des modulations de l'usage de la norme islamique. Ils fonctionnent comme des idéaux-types: le rejet, la nuance, l'acceptation.

Sur le site salafs.com, à la rubrique «banque islamique", une fatwa de Muhammad Nasir Din Al-Albani (1914-1999), théologien albanais, condamne l'usage de la murâbaha: dans le cas de figure où «Un homme veut (contracter un emprunt) auprès d'une banque islamique pour construire (ou acheter) une maison. La banque l'achète pour lui à condition qu'il paie un peu plus cher, et on nomme cela «le gain» (ribh).», Muhammad Nasir Din Al-Albani, répond: «Cette pratique n'est pas propre aux banques dites islamiques, et même d'autres banques peuvent pratiquer cela, et cette pratique n'est rien d'autre qu'une pratique usuraire ${ }^{24}$. Cette position interdit la pratique de ce mode opératoire en raison de sa nature usuraire et trompeuse, «il la nomme par un nom autre que le sien, ils appellent ça un prêt sans intérêts, mais ce n'est pas un prêt sans intérêts ». Pour le théologien, «Le prêt sans intérêts consisterait à ce qu'ils l'achètent 10000 dinars et te prennent 10000 dinars $»^{25}$.

Une position nuancée consiste à reconnaître le caractère controversé de la transaction, en tant qu'instrument de dette, tout en conditionnant son usage car si l'intérêt, ribâ', est interdit, le profit, lui, est encouragé ${ }^{26}$. C'est la position de l'organisme consultatif français, $\mathrm{ACERFI}^{27}$, qui relaye les standards et

24. http://www.salafs.com/modules/news/article.php?storyid=10052. Dernière visite du site le 13 juin 2017.

25. Ibid.

26. Sourate II, verset 275. L'AAOIFI citent également le verset «ce n'est pas un crime pour vous de rechercher la bonté de Dieu» (Sourate Al-Baraqah, verset 198) en interprétant le terme de "bonté " par don, générosité, ce qui apporte un profit et gain.

27. L'Audit, Conformité Et Recherche en Finance Islamique (ACERFI) est un organisme consultatif de conformité islamique français crée en 2008 sous la forme d'une association de la loi 1901. Il œuvre à l'élaboration, au développement et au contrôle des opérations de finance islamique en France et dans le monde francophone. Il existe deux autres comités francophones de conformité islamique, le COFFIS, le Conseil Français de la Finance Islamique, (2009) et le CIFIE, le Conseil Indépendant de la Finance Islamique en Europe, (2010). 
adopte une position proche de celle de l'AAOIFI. Le jurisconsulte Mohamed Patel ${ }^{28}$ estime: "Nul doute que, d'un point de vue comptable et économique, la murâbaha et le prêt à intérêt conduisent à un résultat qui est apparemment similaire. Mais ce n'est pas pour autant que, d'un point de vue juridique, on puisse assimiler ces deux opérations qui ont un objet fondamentalement différent. C'est bien ce qui avait été rappelé par le Coran aux Arabes païens de La Mecque lorsque ces derniers avaient comparé la vente (et le profit qui en résulte) et le ribâ' (intérêt): "Dieu a permis la vente et a interdit le ribâ"» (sourate II, verset 275) » ${ }^{29}$. Le jurisconsulte rappelle que la murâbaha est une transaction commerciale d'achat revente, et non financière. Légitime dans le Coran, elle peut ainsi servir de «financement alternatif» aux musulmans en France qui veulent éviter les usages interdits par le Coran, "ainsi, plutôt que d'octroyer un prêt rémunéré pour financer l'acquisition d'un bien, la banque achète celui-ci et le revend à son client, au comptant ou à crédit, avec une marge de profit définie ${ }^{30}$.

Le Conseil Européen de la Fatwa ${ }^{31}$ "réitère et confirme les avis des différents conseils islamiques de jurisprudence musulmane qui considèrent que les intérêts bancaires sont interdits pour le musulman » ${ }^{32}$. Mais depuis 1999, une décision du Conseil autorise les minorités islamiques vivant en Europe, n'ayant pas accès à des banques islamiques, à recourir à des prêts conventionnels tout en les incitant à trouver d'autres moyens et procédés légaux pour financer des projets personnels ou professionnels ${ }^{33}$. Cette position encourage les musulmans à vivre en conformité avec les prescriptions islamiques tout en conciliant les règles et normes du contexte dans lequel ils vivent ${ }^{34}$. Pour le Conseil, «Les contrats de paiement différé avec

28. Disciple de Taqi Usmani, président de l'AAOIFI, Mohamed Patel est un jurisconsulte réunionnais membre de l'ACERFI. Il est aussi président du sharîa board de la Chaabi banque (France) et membre du sharîa board de 570easi, une entreprise française de courtage islamique.

29. http:/www.finance-muslim.com/2012/07/murabaha-credit. Finance-muslim est le site Internet francophone crée par Mohamed Patel pour «expliquer la finance islamique à tous ", dernière visite le 13 juin 2017. Le shariah scholar a également crée les pages Internet Muslimfr.com et Musulmane.com.

30. http://www.finance-muslim.com/2012/04/presentation_murabaha. Dernière visite le 13 juin 2017 31. Le Conseil Européen de la Fatwa et de la Recherche est une fondation créée en 1997 à l'initiative de l'Union des Organisations islamiques en Europe (UOIE). Son siège est situé à Dublin, elle est dirigée par Yûsuf Al-Qardâwî, également président de l’Union internationale des savants musulmans. Le Conseil a comme objectif de «se donner les moyens de « rester musulmans » tout en vivant dans de nouveaux environnements, fondés sur des rapports au religieux, à la pratique et à la morale, que les musulmans ne connaissaient pas dans leur pays d'origine ", Conseil européen de la Fatwa et de la recherche, Recueil de Fatwas, série n 1 , Avis juridiques concernant les musulmans d'Europe, Éditions Tawhid, 2002. Voir article de Sandra Huout dans ce dossier.

32. Conseil Européen de la Fatwa, Fatwa 26, décision 2-4.

33. Ibid.

34. «Le Conseil incite les musulmans à trouver des alternatives légales qui leur permettent d'accéder à la propriété sans passer par les crédits à intérêts ", et encourage "les associations musulmanes à négocier avec les banques afin de proposer aux clients musulmans des produits satisfaisants ", ibid. 
élévation de prix sont parfaitement valables [la murâbaha], c'est le prix du bien qui change [...] (Mais) si, à l'heure actuelle, les conditions [...] ne sont pas réunies, alors, le conseil, [...] autorise l'achat de maisons par crédit bancaire à intérêt ${ }^{35}$.

Le corpus des avis juridiques relatif aux usages des contrats de financements islamiques est multiple. L'effort de standardisation des procédures licites et d'uniformisation par l'AAOIFI est aussi accompagné d'une diversité d'interprétations des normes et de leurs traductions. Les conditions de leur application dans un contexte spatio-temporel donné montrent cette tension permanente entre l'adaptation à l'environnement immédiat et la recherche d'innovations techniques, sociales qui permettent le dépassement des conflits. «La référence à la charia (sic) prend (alors) des contours plus pragmatiques. Elle devient [...] une norme flexible dont l'économie générale doit être repensée à l'aune du vécu des populations musulmanes en contexte sécularisé» (Frégosi, 2012: 76).

\section{La circulation des normes liées aux transactions islamiques: le cas de la France et de la Grande-Bretagne}

Le droit n'est plus seulement lié à l'État et à son territoire, il devient un lieu de rencontre de référentiels multiples qui fait interagir une multiplicité de répertoires normatifs (Dupret, 2000). La circulation en Europe des normes islamiques liées aux transactions crée des tensions, mais selon les lieux, les conflits ne se cristallisent pas sur les mêmes questions et n'ont pas les mêmes effets disruptifs (Göle, 2013). En Grande-Bretagne, des dispositions législatives ont permis le développement de ce secteur financier mais la question du rôle des sharîa boards dans la gouvernance des institutions financières islamiques, la disparité des avis juridiques ainsi que la prise en compte des normes islamiques en cas de litiges devant les tribunaux ont suscité l'embarras des autorités compétentes. En France, bien que la reconnaissance de ces normes en cas de litige devant les tribunaux ait été établie ${ }^{36}, l^{\prime}$ introduction de dispositions législatives permettant le développement de la finance islamique n'a pu aboutir.

Dans les deux cas, ces dissensions témoignent à la fois de processus de résistance et des arrangements qui par des jeux de répertoires normatifs (positif et islamique) (Dupret, 2000) définissent les contours d'une communauté morale nationale (Fassin, 2012). En France, le profit engendré dans le cadre

\footnotetext{
35. Le Conseil Européen de la Fatwa et de la Recherche s'est fondé sur l'argument stipulant qu'en cas de nécessité absolue, l'interdit devient permis (verset 119 et 145 de la sourate vi) et sur l'avis des Hanafites et Hanbalites qui autorisent les minorités musulmanes à entreprendre des transactions illicites en matière d'affaires sociales, économiques et politiques dans la mesure où le contraire peut porter préjudice aux minorités musulmanes, ibid.

36. «Leur juridicité admise, les règles de la Chari'a (sic) choisies par les parties à un contrat international de financement islamique seront appliquées par les tribunaux français si le différend afférant à ce contrat international vient à leur être soumis directement " Rapport du Groupe de travail sur le droit applicable et le règlement des différends dans les financements islamiques, G. Affaki (dir.), I. Fadlallah, D.Hascher, A. Pézard, F-X. Train, 2009, p. 9.
} 
d'une murâbaha a été assimilé à de l'intérêt ${ }^{37}$ alors qu'en Grande-Bretagne, les magistrats ont écarté toute référence à la sharîa, voire ignoré l'interdiction du ribâ', dans leurs jugements. La réception des acteurs (législateurs et juges) va ainsi dans le sens d'une réitération des monopoles d'usages financiers, juridique ou culturel. Ces résistances sont accompagnées d'efforts de traductions et d'interprétations de la part des acteurs impliqués (législateurs, professionnels, juristes, universitaires, etc.) qui aboutissent à des interpénétrations (Göle, 2005 ) telles que les instructions fiscales en France (2010) ou le Finance Act 2003 en Grande-Bretagne.

La volonté de développer le secteur de la finance islamique en France témoigne de la conciliation entre l'islam, la logique du marché et le politique. Le référentiel islamique devient alors « une ressource politique qui a toute sa place dans la stratégie et la prospective économique de la France» (Frégosi, 2012: 76). Les pouvoirs publics français se sont intéressés au développement de ce secteur à la fin des années 2000. En 2008, Christine Lagarde, ministre de l'Économie, de l'Industrie et de l'Emploi, affirmait la volonté du gouvernement « d'adapter (l') environnement juridique pour (...) bénéficier à la finance islamique ${ }^{38}$. Pour Paris Europlace ${ }^{39}$, acteur de la place financière de Paris, «Dans le contexte (...) de crise de la finance mondiale, l'économie française a plus que jamais besoin de financements (...). La finance islamique, dopée par la rente pétrolière de ces dernières années, offre une alternative pertinente à la finance conventionnelle ${ }^{40}$.

L'intérêt des pouvoirs publics pour la finance islamique est motivé par le financement des infrastructures, les projets de l'État, les collectivités territoriales ou encore les petites, moyennes et grandes entreprises. En ce sens, les mesures entreprises ont visé le développement de fonds d'investissement davantage que d'offres de produits et de services aux particuliers. En 2008, le Sénat français avait organisé un débat sur l'opportunité d'ouvrir le marché national à la banque de détail islamique ${ }^{41}$. Jean François Pons, directeur des relations européennes et internationales de la fédération bancaire de France, s'est référé à la fatwa émise en 1999 par le Conseil européen de la Fatwa ${ }^{42}$

37. Instruction du 23 juillet 2010 relative au régime applicable aux opérations de murâbaha, Direction générale du des Finances Publiques, Bulletin Officiel des Impôts, n 78 du 24 août 2010, 4 FE/S1/10. 38. Christine Lagarde lors des Rencontres Financières Internationales de Paris Europlace, L'environnement juridique de la Place financière de Paris: les principales réformes 2008-2009, Paris Europlace, 2009, p. 12.

39. Paris Europlace est une association en charge de développer la Place financière de Paris. Créée en 1993 par des instances publiques (Banque de France, Caisse des Dépôts, Chambre de Commerce et de l'industrie, Conseil Régional d'Ile-de-France), elle fédère les acteurs de l'industrie financière à Paris (entreprises industrielles et commerciales, banques, sociétés de bourse, sociétés de gestion, associations professionnelles, sociétés de conseil, cabinets d'avocats) et représentent les intérêts de l'industrie financière française auprès des pouvoirs publics et des instances européennes.

40. ibid., p. 56.

41. «La Finance islamique ", Table ronde organisée par la Commission des Finances du Sénat, 14 mai 2008, Paris, France.

42. Conseil Européen de la Fatwa, Fatwa 26, décision 2-4. 
- qui autorise l'usage des crédits bancaires aux musulmans - pour décourager cette proposition qui "conduirait à un "communautarisme bancaire». Il s'appuie sur un élément du répertoire islamique pour dissuader le législateur de développer une offre islamique à l'échelle de la banque de détail. La référence islamique sert ici à perpétuer le modèle universaliste français qui cherche à unifier les différences en réfutant les particularismes.

\section{Des « frottements juridiques» en France}

En 2008, Paris Europlace crée en son sein une «Commission Finance islamique » afin d'accueillir favorablement les investissements islamiques. Dans le cadre de cette commission, un partenariat avec l'AAOIFI est signé à Bahreïn en janvier 2009 en vue de traduire et d'assurer «la promotion des standards Sharia (sic.) de l'AAOIFI en France " ${ }^{43}$. Un groupe de travail sur le droit applicable et le règlement des différends dans les financements islamiques est également créé dans le cadre de cette commission. Il est composé de personnalités du milieu académique et juridique sous la direction de Georges Affaki ${ }^{44}$. Ce groupe de travail devait étudier les modalités d'accueil de la finance islamique en France et plus particulièrement, le droit applicable en cas de différends portant sur les financements islamiques. Le rapport formule des avis dans l'objectif de rassurer les investisseurs qui souhaitent entreprendre en France de manière conforme à l'éthique islamique.

Selon ce rapport, "la partie des règles de la Chari'a (sic) liée aux transactions ( $m u^{\prime}$ âmalât $)$ susceptibles de s'appliquer aux financements islamiques ne révèle pas de contrariétés avec l'ordre public substantiel français » 45 . Certaines modifications du droit français sont nécessaires pour rendre les produits et les opérations financières islamiques au moins aussi coûteuses que les produits financiers conventionnels. Les acteurs parlent de "frottements juridiques » ${ }^{46}$. En septembre 2009, une loi favorisant l'accès au crédit des petites et moyennes entreprises proposée par Chantal Brunel, députée UMP, proposait d'introduire une modification du Code civil «pour permettre l'émission sur la place de Paris de produits compatibles avec les principes éthiques musulmans ${ }^{47}$

43. Communiqué de presse Paris Europlace - Bahreïn Roadshow, Manama, 18 janvier 2009. 44. George Affaki est avocat, membre exécutif et responsable des financements structurés à BNP Paribas et maître de conférence à l'université de Panthéon Assas.

45. ibid., p. 10.

46. Si le régime de marchand de bien semble être adapté aux opérations de murâbaha, la transaction implique une double taxation des droits de mutation en matière d'enregistrement, des taxes notariales, des honoraires du notaire, etc. - à l'achat et à la vente du bien - ce qui augmente le prix d'achat final. Il s'agit de lever cette double taxation et d'adapter le droit français de telle manière à ce que les opérations et produits financiers islamiques soient "compétitifs » sur le marché français.

47. Chantal Brunel, députée UMP de la $8^{\mathrm{e}}$ circonscription de Seine et Marne, lors de la séance à l'Assemblée nationale sur la proposition de loi favorisant l'accès au crédit des petites et moyennes entreprises, 17 septembre 2009. Le régime français de fiducie n'octroyant pas un droit de propriété - obligatoire dans le cas des contrats islamiques - au bénéficiaire de la fiducie, la députée 
en particulier des suk̂ेks ${ }^{48}$. Ce projet de loi a suscité l'opposition de soixante députés qui ont déféré l'ensemble de la loi auprès du Conseil Constitutionnel. Ce dernier a statué «que ces deux articles étaient sans lien avec l'objet initial de la proposition de loi qui tendait à favoriser l'accès au crédit des petites et moyennes entreprises ${ }^{49}$, et les a déclarés contraires à la Constitution.

Le débat qui s'est tenu à la suite de la proposition de loi révèle que dans cette dialectique, les espaces séculiers européens, confrontés au référentiel islamique, sont amenés à réitérer leur singularité par «des opérations d'évaluation et de jugement qui permettent [...] de recomposer (une) communauté morale nationale» (Fassin, 2012). Elles «renvoient à des langages autorisés, à des gammes de règles organisées autour d'un principe légitimateur admis dans le contexte du moment et du lieu. » (Dupret, 2000 : 13). Pour les défenseurs de la loi, majoritairement de l'UMP, « cette modification de la fiducie est tout à fait conforme aux principes traditionnels $\mathrm{du}$ droit civil français » ${ }^{50}$. Ils invoquent la « civilisation chrétienne » - qui pendant longtemps interdisait le prêt à intérêt - pour justifier de la continuité juridique de la proposition de loi. Quant à ceux qui s'y opposent, majoritairement issus du parti socialiste, ils évoquent le principe de la laïcité et le non-respect des valeurs et lois républicaines dès lors que le droit français aurait à adopter des mesures visant la reconnaissance des principes de la sharîa. Henri Emmanuelli, député du parti socialiste, offensé, y voyait « une introduction de la charia (sic) dans le droit français » 51 .

La normalisation juridique des contrats de murâbaha, sukhk ijâra $^{52}$ et istisnấ, 53 'a pas abouti par la voie législative mais par la publication d'instructions fiscales de la Direction générale des Finances Publiques en août 2010. Ces fiches témoignent du «relatif - et transitoire - consensus

proposait à l'Assemblée nationale de modifier l'article 2011 du code civil pour permettre au détenteur de sukûk de se prévaloir d'un droit de propriété des actifs engagés. La modification de l'article impliquerait alors la copropriété des actifs tangibles en conformité aux normes islamiques. 48. Les suk̂uks sont des obligations islamiques adossés à des actifs tangibles, ils se rapprochent du trust en droit anglais et de la fiducie en droit français.

49. Communiqué de presse du Conseil Constitutionnel du 14 octobre 2009.

50. Chantal Brunel, Assemblée nationale, 17 septembre 2009.

51. Discussion suite à la proposition de l'article 6 series B sur «l'adaptation du régime de fiducie pour permettre l'émission d'instruments financiers conformes aux principes de finance islamique», p. 36. 52. L'ijâra est un contrat au terme duquel une entité met un bien à disposition d'un client pendant une durée déterminée, en contrepartie du versement de loyers. Ce contrat peut être assorti d'une promesse de vente ou d'une option d'achat (Direction générale des Finances publiques, instruction fiscale du 23 juillet 2010, 4FE/S3/10). Ijâra signifie également « loyer », il se rapproche des contrats de crédit-bail ou de leasing (opération de location) en finance conventionnelle (Saïdane, 2009). 53. L'istisnâ' est un contrat de financement par lequel un financier, finance pour son compte propre ou pour le compte de son client, la construction d'un ouvrage mobilier ou immobilier auprès d'un tiers qui le construit ( «le fabricant»). Le financier paie le fabricant au comptant ou avec un échéancier durant la phase de construction (Direction générale des Finances publiques, instruction fiscale du 23 juillet 2010, 4FE/S4/10). Cet instrument de financement est un contrat de vente avec livraison différée des marchandises comparable à un contrat à terme cession-bail en finance conventionnelle (Saïdane, 2009). 
(qui) suggère qu'(il) existe une sorte de niveau plus profond d'accord sur les valeurs permettant que, dans un contexte historique donné, certains arrangements moraux soient concevables, qui ne l'étaient pas avant et ne le seront plus après » (Fassin, 2012: 43). On peut également parler ici de plasticité des normes et du droit pour décrire cette caractéristique malléable du droit et la capacité qu'ont les normes d'être manipulées et manipulables selon le contexte d'usage pour trouver à chaque situation, les ressources et les outils qui légitiment (ou non) leurs usages.

À la difficulté de transférer des éléments du répertoire islamique dans le droit français, s'ajoute la difficulté d'interpréter et d'appliquer des normes, des valeurs qui relèvent d'un programme de vérité ${ }^{54}$. Pour la murâbaha, la Direction générale des Finances Publiques a statué que "Sur le plan économique, le Revenu du Financier constitue la rémunération d'un différé de paiement assimilable, sur le plan fiscal, aux intérêts dus durant cette période dans le cadre d'un financement conventionnel. Par conséquent, les règles fiscales doivent être appliquées, pour le Financier, en regardant le Revenu comme un flux d'intérêts que produirait un financement conventionnel équivalent, et pour le Client, comme un flux d'intérêts qu'il acquitterait dans le cadre d'un financement conventionnel équivalent "55. L'administration fiscale a donc fait le choix d'aligner le régime fiscal de la murâbaha sur celui d'un prêt conventionnel, établissant une équivalence entre le profit, licite, et l'intérêt, illicite en islam.

Le transfert de la finance islamique en droit français a conduit à une «acculturation juridique» - «processus suivant lequel les systèmes de normes juridiques sont construits et modifiés par les contacts et les interpénétrations entre cultures et sociétés (Rouland, 1968) (qui) engendrent des modifications susceptibles de changer la nature de l'ensemble des systèmes juridiques en présence.» (Affaki, 2008: 159). Les mesures prises par l'administration fiscale - allégement des modalités d'imposition du profit financier, exonération des retenues à la source pour les étrangers et limitation des droits d'enregistrements lors de l'achat du bien (régime des marchands de biens) - introduisent le répertoire islamique dans le droit français mais cela, à travers l'emprunt des procédés conventionnels qui vont à l'encontre des prescriptions et principes de la finance islamique.

54. Pour Baudouin Dupret, «le jeu des répertoires juridiques positif et islamique, [...], procède d'idées et de logiques idéelles (représentations et visions du monde) constituées hiérarchiquement en programmes de vérité (ensembles de croyances)»(Dupret, 2000: 326). En ce sens, les normes sont construites et reproduisent des valeurs intrinsèquement liées à des systèmes de pensée, leur appréciation nécessite l'adoption sinon l'empathie, le Verstehen, de la représentation du monde et de l'ensemble des croyances qui transcendent ce système de pensée.

55. Instruction du 23 juillet 2010 relative au régime applicable aux opérations de murâbaha, Direction générale du des Finances Publiques, Bulletin officiel des Impôts, nº 78 du 24 août 2010, $4 \mathrm{FE} / \mathrm{S} 1 / 10$. 


\section{Les sharîa boards en Grande-Bretagne, des instances consultatives et non décisionnaires}

En Grande-Bretagne, les premiers produits bancaires de détails islamiques sont introduits dans les années 1990 par des banques du Moyen-Orient et de l'Asie du Sud Est, mais la plupart ne sont alors pas contrôlés par les autorités britanniques - la Financial Services Authority ${ }^{56}$ et la Banque d'Angleterre. La croissance du marché a incité ces instances à étudier les obstacles financiers et juridiques de ce secteur. Quatre réformes législatives ont été adoptées ${ }^{57}$ dont le Finance Act 2003 qui prend en compte la contrainte de l'interdiction de l'intérêt et met fin à la double imposition à l'occasion des doubles transferts de propriété. Il prévoit ainsi l'exonération de la Stamp Duty and Land Tax (SDLT) pour la deuxième partie de la vente à un particulier qui consent une hypothèque ${ }^{58}$.

Si les normes fiscales ont été adaptées, le statut et le rôle des sharîa board dans la gouvernance bancaire ont occasionné des réserves. La conformité des transactions à la sharîa n'a de valeur que si elle est certifiée - sous forme de fatwa - émises par le sharîa board de l'institution financière; ce faisant, la finance islamique introduit une instance religieuse de certification au sein des institutions financières. Dans son rapport, la Financial Service Authority souligne la difficulté de contrôler cette instance dans la gouvernance de la banque. Elle exige que les institutions financières islamiques souhaitant s'installer en Grande-Bretagne considèrent le sharîa board comme instance consultative et non pas comme instance décisionnaire.

Cette approche s'illustre dans la prise en compte des avis des jurisconsultes par les juges anglais en cas de litige: réduits au statut d'expertise, l'avis des sharîa scholars est peu utilisé en tant que référence dans leurs jugements. En France, le groupe de travail sur le droit applicable et le règlement des différends dans les financements islamiques, a statué que «les fatawa (sic) délivrées par les conseils de supervision de Chari'a (sic) (Shari'a(sic) boards comités composés de jurisconsultes ayant compétence pour se prononcer sur la validité des transactions financières des établissements financiers au regard des normes de la Chari'a (sic) - constitueront des précédents potentiellement applicables aux différends en cours " ${ }^{59}$. Ces avis juridiques ainsi que les normes standardisées de l'AAOIFI peuvent donc servir de référence au juge ou à l'arbitre en cas de litige.

56. La Financial Service Authority est un organisme non gouvernemental financé par les entreprises du secteur financier et responsable devant le Ministère du Trésor et le Parlement. Créée en 1997, suite à l'externalisation de la supervision bancaire dont la Banque d'Angleterre était jusque là responsable, elle est en charge depuis les années 2000 de la réglementation des hypothèques (2004) et des assurances (2005).

57. Michael Ainley, Ali Mashayekhi, Robert Hicks, Arshadur Rahman, Ali Ravalia, Islamic Finance in the UK: regulation and challenges, Financial Services Authority, 2007.

58. Finance Bill 2003, resolution 20, clause 72.

59. Ibid., p. 22. Ce rapport reconnaît au juge ou à l'arbitre la possibilité de se référer au répertoire islamique - en particulier, aux normes standardisées établies par l'AAOIFI - en cas de litige. 


\section{L'utilisation du référentiel juridique islamique en cas de litige devant les tribunaux britanniques}

La présence des musulmans en Europe tout comme leur inscription dans la globalisation amènent de plus en plus « les États du monde occidental [...] (à se confronter) à des problématiques juridiques et des revendications qui mettent en jeu une normativité islamique» (Bras, 2012: 287). Avec la pénétration de la finance islamique en Europe et l'usage de modes de financements conformes à l'éthique islamique, les tribunaux civils européens ont été plus récemment engagés à juger de la conformité des transactions utilisant un référent islamique. Ces litiges supposent des jugements - voire des sanctions - établis par des autorités légales non islamiques dans des juridictions non islamiques, ce qui interroge la possibilité d'un pluralisme juridique (Bras, 2012) et de la coexistence, voire de l'articulation d'ordres normatifs et de savoirs multiples (Dupret, 2000). En particulier, interagissent ici un répertoire positif, séculier et un répertoire islamique sans tradition juridique en Europe.

Dans cette interaction, le paradigme dominant procède-t-il à des ajustements qui conduisent à la restructuration des catégories conceptuelles? Ou bien ignore-t-il les éléments qui lui sont étrangers et conduit-il à une clôture référentielle du répertoire dans lequel le droit est appliqué? (Dupret, 2000). En miroir des travaux de Baudouin Dupret en Égypte, une radiographie de la pratique interprétative judiciaire britannique permet d'observer les stratégies que les magistrats anglais adoptent face au répertoire islamique et des effets qui en résultent. Elles prennent la forme de l'évitement - le juge se dispense de prendre en considération les règles de droit islamique en se référant à des normes nationales ou communautaires - du contournement - le juge préfère revenir aux catégories du droit qu'il connaît (Bras, 2012) et de l'entendement où le juge se prononce et raisonne selon les catégories de droit qu'il connaît, mais admet les limites de son savoir, la pluralité des répertoires normatifs et la multiplicité des programmes de vérités.

Les litiges exposés montrent la difficulté des juges anglais à interpréter la normativité islamique. S'ils sont sollicités pour juger de la conformité de contrats islamiques, ils se trouvent amenés à statuer sur la légitimité et l'usage de la sharîa devant les tribunaux anglais ${ }^{60}$. Chaque cas soulève la question de la légitimité de l'autorité compétente pour statuer en cas de litige engageant une normativité islamique. Cette légitimité ne se limite pas à l'exercice de l'autorité, elle concerne aussi la connaissance du répertoire islamique et, plus

60. On pourrait rapprocher cette stratégie à celle de la «substantialisation » décrite par Baudouin Dupret, néanmoins il n'est pas question ici d'interpréter les dispositions du droit positif anglais portant sur la religion comme l'exercice des cultes ou encore la liberté de conscience, par exemple le choix des parties de se référer au répertoire islamique dans un contrat de financement islamique. Il ne s'agit pas non plus de «survalidation" dans la mesure où les juges ne se servent pas du répertoire islamique pour conforter la loi positive (Dupret, 2000). Il s'agit d'interroger les jugements énoncés par des juges anglais en cas de litiges faisant référence au répertoire islamique. 
largement, la multiplicité des programmes de vérités. Les différentes stratégies employées par les magistrats anglais témoignent d'une hiérarchie des répertoires normatifs - tant dans l'usage des référentiels que des autorités qui les énoncent - qui conduit à une clôture référentielle (Dupret, 2000).

\section{Méconnaissance et évitement: l'affaire Symphony Gems vs. l'Islamic Investment Company of the Gulf}

L'affaire Symphony Gems ${ }^{61}$ opposait l'Islamic Investment Company of the Gulf Ltd. (IICG), une institution financière saoudienne dont le siège se trouve aux Bahamas, à un diamantaire belge, Symphony Gems N.V. L'objet du litige portait sur la conformité à la sharîa d'une murâbaha qui liait les deux parties dans le cadre du financement d'un achat de pierres précieuses. L'IICG devait acheter les pierres précieuses à Precious Ltd, une société localisée à Hong Kong, puis les revendre à Symphony Gems avec une marge de profit consentie et des facilités de paiement ${ }^{62}$. En février 2000, l'IICG avait procédé au paiement de la marchandise dans sa totalité sur le compte de Precious Ltd sans qu'aucun paiement n'ait été encore fait par Symphony Gems. Precious Ltd n'avait pas non plus livré la marchandise ni procédé au transfert de l'acte de propriété à l'IICG ou à Symphony Gems ${ }^{63}$.

L'IICG étant une société d'investissement saoudienne, les avocats représentant la défense de Symphony Gems ont demandé à la Cour Commerciale de la Queen's Bench Division à Londres d'invalider le contrat selon les lois islamiques saoudiennes. Le juge Tomlison a déclaré la requête invalide car le siège de la société d'investissement se trouvait au Bahamas où s'applique la loi britannique d'autant que le contrat indiquait le droit anglais dans la clause d'applicabilité. Il a ignoré la décision des sharîa scholars appelés en tant qu'experts qui avaient conclu que le contrat n'était pas conforme à une murâbaha, et rejeté l'argument de la non-possession des biens en raison du fait que cette condition n'était pas un prérequis mentionné dans les clauses du contrat ${ }^{64}$. Le juge a interprété les accords commerciaux de manière littérale (Moghul et Ahmed, 2003) et statué en faveur de la banque selon les lois

61. Islamic Investment Company of the Gulf (Bahamas) Ltd vs. Symphony Gems NV and others, per Tomlison J., [2002] EWHC 1 (Comm).

62. Dans une murâbaha, l'acheteur final peut refuser d'acheter le bien selon l'International Islamic Fiqu Academy, resolution n ${ }^{\text {os }} 40-41$ (2/5 et 3/5). Il peut être stipulé en cas d'annulation de la vente, que l'acheteur final paie une compensation à l'intermédiaire financier qui correspond à la différence du prix d'achat convenu et du prix de revente, mais cette clause doit être spécifié dans le contrat. Standard no 8, Shari'a standards for Islamic financial institutions, AAOIFI, 2010 (1432H), p. 132.

63. Contrairement à ce qui prévaut en finance conventionnelle, dans une transaction de $m u$ râbaha, l'institution est un intermédiaire marchand qui est propriétaire du bien le temps de la transaction. Cette condition de propriété s'appuie sur un hadith qui ordonne: «ne vends pas ce qui ne t'appartient pas ", rapporté par Al- Tirmidhi (Sunan al-Tirmidhi 3/534) et par Al-Tabrani (al-Mu'jam al Awsat 5/66), Dar al Haramayn, Cairo, 1415H.

64. Clauses $4.3 ; 4.4 ; 5.1 ; 5.6 \mathrm{du}$ dit contrat. 
britanniques en vigueur - tel qu'il était mentionné dans la clause d'applicabilité juridique - en imposant des compensations de retard ${ }^{65}$.

Le magistrat adopte dans cette affaire la posture de l'évitement: il se réfère au droit anglais et ne prend en considération ni les normes ni l'avis des jurisconsultes islamiques. Son interprétation littérale des clauses du contrat lui permet d'éviter d'avoir à interpréter selon le paradigme islamique. Il ignore ainsi deux aspects fondamentaux d'un contrat islamique: l'obligation de possession de biens et l'interdit du ribâ'. Au-delà de la question d'une hiérarchie normative, on peut se demander si le jugement aurait été similaire, si le magistrat connaissait certains éléments du répertoire islamique.

\section{Cécité et contournement : l'affaire Beximco vs. Shamil Bank}

L'affaire Beximco ${ }^{66}$ opposait une banque islamique, Shamil Bank de Bahreïn E.C., localisée à Bahreïn au groupe Beximco, localisé au Bengladesh. L'objet du litige en première instance portait sur la conformité de deux contrats de murâbaha ${ }^{67}$ - certifiés par le sharîa board de la banque - et d'un contrat d'ijâra établis entre les parties dans le cadre d'un financement du fonds de roulement de la Beximco Pharmaceuticals Ltd. Quatre ans après la signature des accords, la Beximco Pharmaceuticals Ltd et la Bengladesh Export Import Co Ltd n'avaient pas payé la totalité de la somme convenue entre les deux contrats de murâbaha et la Shamil Bank n'avait pas acheté les produits décrits dans les contrats. Après accord entre les parties, la banque et les deux entreprises ont accepté d'annuler un certain montant de la somme impayée en échange de l'acquisition de titres de propriété d'actifs de l'entreprise ${ }^{68}$.

65. L'AAOIFI autorise le paiement en différé, mais il est interdit d'ajouter des pénalités pour retard de paiement considérées comme du ribâa'. Le fait de ne pas rembourser sa dette si l'on a la capacité de le faire, est haram, illicite ; si le débiteur a fait banqueroute ou n'est plus dans la capacité de rembourser le créditeur, ce dernier dans le cadre d'une murâbaha, peut vendre le/ les actifs qui font l'objet de la transaction à un autre acheteur (Shari'a standard $n^{\circ} 3$ Default in Payment by a Debtor, p. 33). Un dépôt de garantie (hamish jeddiyyah) peut également être engagé. À la fin de la transaction, il doit être déduit du prix final de la vente ou rendu à l'acheteur final. Des arrhes peuvent également être engagées, en cas de défaut de paiement, la totalité du montant revient à l'institution (Shari'a standard n'8 Murabaha to the Purchase Orderer, p. 111-136).

66. L'affaire Beximco a fait l'objet de deux jugements par deux instances, la High Court of Justice Queen's Bench Division en première instance en août 2003 et la Supreme Court of Judicature Court of Appeal (civil division) en seconde instance en janvier 2004. Shamil Bank of Bahrein EC vs. Beximco Pharmaceuticals Ltd and others, Morison J., pour la décision de la Commercial Court du $1^{\text {er }}$ août 2003 [2003] EWHC 2118 (Comm), [2003] 2 All ER (COMM) 849, et Potter L.J. pour l'arrêt de la Court of Appeal du 28 janvier 2004 [2004] EWCA Civ 19,[2004] 4 All ER 1072 .

67. Les contrats de murâbaha stipulaient que l'institution financière acceptait d'acheter des biens spécifiés dans le contrat par l'intermédiaire de la Bengladesh Export Import Co Ltd et les revendrait immédiatement à la Beximco Pharmaceuticals Ltd.

68. Cet accord a été établi sous la forme d'une ijâra dans lequel la banque louait ses titres à l'entreprise en échange d'un paiement de frais d'utilisation. 
Contrairement à Symphony Gems, dans lequel le contrat ne désignait que les lois britanniques comme droit applicable au contrat, dans le cas de Shamil bank, la clause d'applicabilité combinait les principes de la sharîa au droit anglais, le contrat mentionnait: "Sous réserve des principes de la sharîa, cet accord doit être régi et construit en accord avec le droit anglais ${ }^{69}$. La cour n'a pas eu à considérer la conformité du contrat aux normes islamiques, elle a rejeté la dualité des référentiels juridiques dans l'exécution du contrat ${ }^{70}$. Pour appuyer son argument, elle s'est référée à la Convention de Rome qui requiert l'application d'un seul système juridique à un contrat ${ }^{71}$ et stipule que ce système doit être lié à un État ${ }^{72}$. Le droit anglais ayant été défini comme cadre juridique, il prévalait donc sur des lois non nationales. L'invocation générale aux principes de la sharîa ne lui permettant pas de statuer sur la validité ou l'invalidité du contrat, le juge a adopté la posture du contournement et préfère revenir aux catégories du droit qu'il connaît, qu'elles soient nationales ou internationales.

Cela avait déjà été le cas en 2000 dans l'affaire Sanghi Polyesters Ltd. (India) v. The International Investor KCFC (Kuwait) ${ }^{73}$ qui concernait un contrat d'istisnâa'. Une clause stipule qu'en cas de litige, le différend est "régi par le droit anglais, sauf s'il entre en conflit avec la sharîa, qui prévaut " ${ }^{74}$. Le juge n'a pas invalidé le contrat car il n'y avait aucune irrégularité ou injustice au regard du droit anglais; pour lui la seule manière de le rendre caduque ne pouvait se faire que par la loi islamique. Dans son argumentaire, il a refusé de se prononcer sur le statut de la sharîa, en admettant qu'il lui était difficile de définir ce qu'est la sharîa (une loi étrangère, un code, un ensemble de coutumes) n'ayant pas les «matériels » suffisants pour. Il conclut en affirmant la supériorité du droit anglais sur le répertoire islamique en rattachant les normes légitimes au territoire dans lequel le jugement est énoncé: «Je souligne simplement l'évidence suivante: quelle qu'elle soit, la sharîa n'est pas le droit de l'Angleterre et du Pays de Galles » ${ }^{75}$.

69. Traduction de "Subject to the principles of the Glorious Sharia'a (sic), the agreements should be governed and construed in accordance with English Law".

70. La Cour a estimé que l'usage de l'expression “Glorious Sharia'a” (sic) ne faisait pas référence à un système normatif désigné dans l'application du contrat, mais se référait aux principes généraux selon lesquels la banque islamique conduisait ses affaires.

71. Convention de Rome ou "Convention sur la loi applicable aux obligations contractuelles ", 80/934/CEE, Rome, juin 1980.

72. Convention de Rome, article 7 «Lois de police», 80/934/CEE, Rome, juin 1980. Le règlement communautaire $n^{\circ}$ 593/2008 de la Convention Rome I modifie cet article en reconnaissant aux parties la possibilité de se référer dans leur contrat à un droit non étatique ou une convention internationale.

73. Sanghi Polyesters Ltd. (India) vs. The International Investor KCFC (Kuwait), per D. Mackie, [2000] Queen's Bench Division Commercial Court.

74. Traduction de: "governed by the Law of England except to the extent it may conflict with Islamic Shari'ah (sic), which shall prevail".

75. Traduction de: "I merely point out the obvious that whatever Shari'a law may be it is not the law of England and Wales". 


\section{Doute et entendement : l'affaire Blom Development vs. Investement Dar Company}

Dans le cas de The Investment Dar Company KSCC vs. Blom Developments Bank $^{76}$, les juges ont statué en première et en seconde instance en faveur de la banque libanaise Blom Developments Bank pour le remboursement de sommes allouées dans le cadre d'un contrat wakâla ${ }^{77}$ établi avec l'Investment Dar Company, une société d'investissement islamique koweitienne. Ce contrat, qui désignait le droit anglais comme droit applicable, avait été certifié par le sharîa board de la société d'investissement. Il stipulait que les fonds devaient être investis dans des opérations respectant la sharîa, conformément à ses statuts, et que chaque trimestre, la société d'investissement devait transférer les bénéfices des investissements à la banque. En 2008, la société d'investissement n'ayant pas pu payer les bénéfices de son investissement, la banque libanaise avait saisi le tribunal de la haute Cour de Justice pour le remboursement de son investissement.

La société d'investissement a plaidé l'invalidité du contrat en raison du fait que le paiement des bénéfices échelonné sur le temps était considéré comme de l'intérêt, ribâ'. En première instance et en seconde instance, le juge a statué en faveur de la banque libanaise, mais contrairement aux autres affaires, il a consenti que le contrat de wakâla pût être "un dispositif pour permettre [...], le paiement des intérêts sous une autre forme, ce qui finalement indirectement est une pratique non conforme à la sharîa». Et de conclure: "il n'est pas approprié pour moi de revoir en détail les preuves des experts, je suis convaincu que je ne peux pas trancher " ${ }^{78}$. Tout en reconnaissant la légitimité du sharîa board de la société d'investissement dans l'appréciation de la conformité du contrat il admettait ne pas posséder l'expertise - la connaissance - pour juger de la conformité d'un contrat islamique.

\section{De la coexistence des deux répertoires (islamique et positif) en Grande-Bretagne et en France}

Ces affaires montrent la difficulté des magistrats anglais à se prononcer sur des normes islamiques: la référence à la sharîa (normativité islamique) devient même obsolète. Ils s'appuient sur des références juridiques nationales ou internationales et ce faisant, inscrivent les opérations d'évaluation et de jugement dans un cadre

\footnotetext{
76. The Investment Dar Company KSCC v Blom Developments Bank SAL (Rev 1) [2009] EWHC 3545 (Ch).

77. La wakâla est un accord dans lequel un des partenaires mandate le second (al wakîl) pour réaliser des investissements en son nom.

78. Traduction de: "albeit difficult to apply, namely, that where one finds, as one does in this master wakâla contract, a device to enable what would at least to some eyes appear to be the payment of interest under another guise, that is at least an indirect practice of a non-Sharia compliant activity. I do not think it appropriate for me to go through the expert evidence in detail because I am satisfied that I cannot resolve which expert is correct on this application", the Investment Dar Company KSCC v Blom Developments Bank SAL (Rev 1) [2009] EWHC 3545 (Ch).
} 
référentiel national. Les stratégies qu'ils emploient ont pour effet l'hégémonie du répertoire positif sur le répertoire islamique et conduisent à une clôture référentielle (Dupret, 2000) qui se traduit par «la représentation d'une normalité culturelle, celle de la tradition authentique que la société considérerait comme seule légitime» (Dupret, 2000 : 208), la pratique de l'intérêt par exemple.

Au-delà de l'observation d'une hiérarchie de normes, ces cas de litiges révèlent un double conflit de légitimité: celui de la référence au répertoire islamique en cas de litige porté devant une cour nationale européenne et celui de l'expertise du magistrat en matière de transactions islamiques pour juger de la conformité des contrats islamiques. Le problème de légitimité n'est pas ici seulement lié à l'autorité - modes et instances légitimes à statuer en cas de litiges qui impliquent une normativité islamique - mais également à la maitrise de savoirs, de connaissances et de techniques qui permettraient de juger des litiges engageant une normativité islamique.

Il en résulte un hiatus d'entendement (Moghul et Ahmed, 2003) qui conduit le plus souvent les juges à écarter la référence islamique de leurs jugements et à ignorer l'avis des experts. Les stratégies employées par les juges sont intrinsèquement liées aux programmes de vérités qu'ils ont tendance à reproduire car elles déterminent non seulement leurs perceptions mais orientent aussi leurs actions (Dupret, 2000): il semble en effet difficile de concevoir le caractère illicite de la pratique de l'intérêt là où elle constitue un monopole d'usage. Plus encore, le magistrat a tendance à reproduire la pratique d'usage dans le cadre de son système de référence, en imputant des "dommages et intérêts » comme le montre l'exemple de l'affaire Symphony Gems.

En France, bien qu'il n'y ait pas encore eu de cas de litiges portés devant un tribunal, le groupe de travail sur le droit applicable et le règlement des différends dans les financements islamiques a statué que «les règles de la Chari'a (sic) relatives (aux transactions) et aux financements islamiques sont des règles de droit (et) doivent à ce titre recevoir effet par les tribunaux français lorsque la Chari'a (sic) vient à être choisie par les parties pour régir leur contrat de financement international $\gg{ }^{79}$. Ce rapport explicite l'applicabilité du répertoire islamique devant les tribunaux français. Un comité d'experts ou sharîa board peut ainsi être sollicité par les tribunaux français pour donner son avis en cas de litige lié aux contrats de financement islamique.

$$
*
$$

La différence islamique relative aux transactions économiques et financières s'observe par des prescriptions issues de la sharîa - Coran et Sunna - réparties dans un répertoire composé de multiples références susceptibles d'être employées. En Europe, ce référentiel entre en confrontation avec les monopoles

79. Ibid., p. 9. 
d'usages financiers, linguistiques, juridiques de la finance conventionnelle, tel que l'usage de l'intérêt ou la spéculation par exemple. Son application s'appuie sur la coopération d'acteurs musulmans et séculiers pour créer les conditions de la genèse d'un secteur financier islamique en Europe. Les pouvoirs publics sont ainsi amenés à réglementer les opérations islamiques et les tribunaux civils sont sollicités pour interpréter et juger de leur conformité à la sharîa. Dans cette sociogenèse les deux acteurs - musulmans et non musulmans - font l'effort d'une réflexion normative pour permettre la traduction et l'adaptation réciproque aux contraintes prescrites. La plasticité de la norme - islamique ou positive - permet alors de dépasser les conflits.

Cette plasticité peut aussi servir à délimiter les frontières d'une communauté morale nationale en réitérant les monopoles d'usage, les valeurs et les normes légitimes et écarter toute référence au répertoire islamique. Les acteurs s'appuient alors soit sur le répertoire positif (lois nationales, conventions internationales) soit sur des valeurs (la laïcité, la «civilisation chrétienne») ou encore sur des références islamiques qui distancient ou rapprochent l'islam de la communauté nationale, mais qui d'une manière ou d'une autre le positionnent dans l'altérité. Le jeu des répertoires positif et islamique montre qu'à «cette pluralité, qui [...] n'implique aucun pluralisme, répond la prétention hégémonique des répertoires »(Dupret, 2000: 317). Sans pour autant créer l'enfermement: "Si la charia (sic) peut être une source d'inquiétude, elle peut tout autant, dans certains cas, devenir une ressource symbolique, voire économique» (Frégosi, 2012: 74-75) et pénétrer les lieux de droit (Dupret, 2000) européens.

Bien que la finance islamique ne montre pas de rupture substantielle avec la finance conventionnelle - comme le serait le troc dans la mesure où il évince tout rapport à l'argent et au profit financier - la forme qu'elle revêt - la rhétorique islamique, les techniques contractuelles et leurs certifications par des sharîa boards des institutions financières - atteste de sa distinction par ses modes opératoires, ses manières de faire et son programme de vérité. Ainsi, "la finance islamique peut sembler, particulièrement en période de crise financière [...], être un moyen de redonner une dimension morale au capitalisme financier» (Chapellière, 2009:15). Pourtant, le défi de son interpénétration ne réside pas dans sa capacité à transformer le capitalisme mais dans la coexistence de valeurs, de pratiques plurielles, et d'un modus vivendi qui suppose un faire ensemble dans le respect des différences.

Bochra KAMMARTI

Centre d'études sociologiques et politiques Raymond Aron (CESPRA) bochra.kammarti@gmail.com 


\section{Bibliographie}

AfFAKI Georges, 2008, «L'accueil de la finance islamique en droit français: essai sur le transfert d'un système normatif ", Laramée J-P. (éd.), La finance islamique à la française. Un moteur pour l'économie, une alternative éthique, Secure Finance, p. 145-172.

Bras Jean-Philippe, 2012, «Des métamorphoses de la charia », Dupret B. (éd), La charia aujourd'hui. Usages de la référence an droit islamique, Paris, La Découverte, coll. «Recherches », p. 279- 291.

Calder Norman, 1998, "Sharîa", Encyclopédie de l'islam, Tome IX, Leiden Brill, p. 331- 338 .

Chapellière Isabelle, 2009, Éthique et finance en Islam, Paris, Koutoubia.

CHAPRA Umer, 2006, "The Nature of Riba in Islam", The Journal of Islamic Economics and Finance, 2, 1, p. 7-25.

De Courcelles Dominique, 2012-2013, « Humanisme spirituel et éthique économique: l'islam pour une finance participative », Revue Études en économie islamique, 6, 1, p. 83-110.

Мoнammed Ali Amir Moezzi (éd.), 2007, Dictionnaire du Coran, Paris, Robert Laffont, coll. «Bouquins».

DUPRET Baudoin, 2000, Au nom de quel droit: répertoires juridiques et référence religieuse dans la société égyptienne musulmane contemporaine, Maison des Sciences de l'Homme/CEDEJ/LGDJ, coll. «Droit et société».

-, 2014, La charia. Des sources à la pratique, un concept pluriel, Paris, La Découverte, coll. «Cahiers libres».

Ebnou Beddy, 2014, Qu'est-ce que la Shari'a ? Introduction à l'épistémologie de l'herméneutique normative en islam, Paris, Le Scribe l'Harmattan, IESE, coll. "Citoyenneté et pluralité ».

Fassin Didier, 2012, "Vers une théorie des économies morales », Fassin D., Eideliman J-S. (éds.), Économies morales contemporaines, Paris, La Découverte, coll. «Recherches », Bibliothèque de l'Iris, p. 19-47.

FrÉGOSI Franck, 2012, "Usages sociaux de la référence à la charia chez les musulmans d'Europe ", Dupret B. (éd.), La charia aujourd'hui. Usages de la référence au droit islamique, Paris, La Découverte, coll. «Recherches ", p. 65-77.

GöLE Nilüfer, 2005, Interpénétrations. L'islam et l'Europe, Paris, Galaade.

-, 2011, “Thinking Islamic Difference in pluralistic Democracies”, Raube K., Sattler A., Mestern S. (eds.), Difference and Democracy. Exploring Potentials in Europe and Beyond, Hambourg, Campus Verlag, p. 159-181.

-, 2013, «La visibilité disruptive de l'Islam dans l'espace public européen: enjeux politiques, questions théoriques ", Cahiers Sens public, 15-16, 1, p. 165-184.

GuÉRANGer François, 2009, Finance islamique: une illustration de la finance éthique, Paris, Dunod, coll. «Marchés financiers".

KAMmARTi Bochra, 2015a, "Éthiques et usages de l'argent: le prêt à intérêt en Islam et en Chrétienté », De Courcelles D. (dir.), Actes pour une économie juste, Paris, Lemieux, p. 149-155.

-, 2015b, «L'interpénétration européenne de la finance islamique », Göle Nilüfer (éd.), En-quête de l'islam européen, Éditions Halfa, p. 168-190. 
Martens André, 2001, «La finance islamique: fondements, théorie et réalité », L'actualité économique, Revue d'Analyse économique, 77, 4, p. 475-498.

MARTIN Virginie, 2012, "La finance islamique: un nouveau pas vers une finance éthique? ", Revue Gérer et Comprendre, 108, p. 15-26.

MAurer Bill, 2002, “Anthropological and accounting knowledge in Islamic banking and finance: rethinking critical accounts”, Journal of the Royal Anthropological Institute, 8, 4, p. 645-667.

Moghul Umar F., Ahmed Arshad A., 2003, “Contractual Forms in Islamic Finance Law and Islamic Inv. Co. of the Gulf (Bahamas) Ltd. v. Symphony Gems N.V. \& Ors.: A First Impression of Islamic Finance”, Fordham International Law Journal, 27, 1, p. 150-194.

Ould Sass Mohamed Bachir, 2010, «Les comités de la Charia: historique, constitution et pouvoir », http://www.acerfi.org/user-res/fichiers/Article_Mohamed_BAchir_Le_ SB\%5B2010\%5D.pdf

Roux Michel, 2012, «Finance éthique, finance islamique: Quelles convergences et potentialités de développement dans la banque de détail française ? ", La revue des Sciences de gestion, 255/256, 3, p. 103-109.

Ruimy Michel, 2008, La finance islamique, Québec, Arnaud Franel, coll. «Finance d'aujourd'hui".

SAEED Abdullah, 1996, Islamic Banking and Interest: a Study of the Prohibition of Riba and its Contemporary Interpretation, Leiden, Brill.

SAÏDANE Dhafer, 2009, La finance islamique à l'heure de la mondialisation, préface Arnaud de Bresson, Paris, Revue Banque, coll. «Les essentiels de la banque».

Usmani Taqi, 1998, An Introduction to Islamic Finance, Idaratul Ma'arif.

\section{Des normes financières islamiques et de leurs circulations en France et en Grande-Bretagne}

Cet article analyse la sociogénèse $d u$ champ financier islamique en France et en Grande-Bretagne à travers l'élaboration, la répartition, la circulation et l'utilisation des normes liées aux transactions islamiques. Cette sociogenèse révèle les dynamiques de créations sociales par l'interpénétration sémantique, juridique et sociale de référents islamiques, tout comme les résistances liées à l'apparition de ces référents en contextes nationaux séculiers. La dissémination des normes islamiques confronte les monopoles d'usages et de pensées européens, se dessine alors des frontières morales aux communautés nationales tout en les renouvelant.

Mots-clés: finance islamique, Europe, économies morales. 


\section{Islamic financial norms and their circulation in France and Great Britain}

This article analyses the social genesis of the Islamic financial sector in France and Great Britain through the elaboration, dissemination, circulation and use of Islamic norms related to financial and economical transactions. This social genesis reveals forms of resistance from the national secular State that shape not only the boundaries of moral communities, but also the dynamic creation of the semantic, legal and social interpenetration of Islamic referents in Europe.

Keys words: Islamic finance, Europe, moral economy, France, UK.

\section{Normas financieras islámicas y su circulación en Francia y Gran Bretaña}

En este artículo se analiza la sociogénesis de la finanza islámica en Francia y en Gran Bretaña a través la elaboración, la circulación y el uso de las normas islámicas relacionadas con la finanza. Esta sociogénesis revela unas dinámicas de creaciones sociales por la interpenetración semántica, jurídica y social de las referencias islámicas, como las resistencias relacionadas con la aparición de estos referentes en contextos seculares. La circulación de normas islámicas enfrenta los monopolios de usos y de pensamientos europeos, asi se dibujan unas fronteras morales a las comunidades nacionales, mientras se regeneran. Palabras claves: finanza islámica, Europa, economias morales. 